\section{Mensurando a demanda física no trabalho: estrutura fatorial e confiabilidade de itens sobre posturas, manuseio de carga e repetitividade}

\author{
Measuring work-related physical demand: \\ factorial structure and reliability of items on \\ posture, handling of loads and repetitiveness

\section{Evaluando los requerimientos físicos en el trabajo: estructura factorial y confiabilidad de ítems sobre posturas, manipulación de carga y repetitividad}

Rita de Cássia Pereira Fernandes 1

Livia Paraguai Cunha 1

Verônica Maria Cadena Lima 1

Kionna Oliveira Bernardes Santos 1

doi: 10.1590/0102-311X00123218

\title{
Resumo
}

O objetivo foi analisar a dimensionalidade de um conjunto de itens que mensura demanda física no trabalho, sua consistência interna e confiabilidade teste-reteste. Realizou-se análise fatorial exploratória de 10 itens de demanda física em duas populações, totalizando 1.070 trabalhadores da indústria de calçados e da limpeza urbana, utilizando-se como método de estimação a média de quadrados mínimos ponderados e variação ajustada, com rotação obliqua Promax. A consistência interna foi medida pelo alfa de Cronbach. $O$ teste-reteste utilizou uma subamostra de 121 trabalhadores, com a utilização do indice kappa de ponderação quadrática. Identificaram-se duas dimensões na limpeza urbana e três na fabricação de calçados: (1) trabalho dinâmico com posturas anômalas; (2) trabalho que implica manuseio de material; $e$ (3) trabalho com exigência de movimentos repetitivos. A confiabilidade foi excelente para postura de trabalho em pé, boa para andando, moderada para levantamento de carga, trabalho agachado, tronco rodado e apenas regular para movimentos repetitivos e braços acima dos ombros. As dimensões identificadas na análise fatorial são compatíveis com a demanda física na limpeza urbana e na indústria de calçados e, em geral, mostraram boa consistência interna. A confiabilidade dos dez itens foi compativel com a literatura, mostrando de excelente a boa confiabilidade para postura geral, moderada para postura de segmento específico do corpo e regular para repetitividade. Resultados revelam especificidades de cada grupo ocupacional, o que sugere dimensionalidade dos itens de demanda física, apoiando o seu uso para a mensuração da exposição física.

Análise Fatorial; Reprodutibilidade dos Testes; Carga de Trabalho;

Inquéritos e Questionários

\author{
Correspondência \\ R. C. P. Fernandes \\ Programa de Pós-graduação em Saúde, Ambiente e Trabalho, \\ Faculdade de Medicina da Bahia, Universidade Federal da \\ Bahia. \\ Largo do Terreiro de Jesus, Centro Histórico, Salvador, BA \\ 40026-010, Brasil. \\ ritafernandes@ufba.br \\ 1 Programa de Pós-graduação em Saúde, Ambiente e Trabalho, \\ Universidade Federal da Bahia, Salvador, Brasil.
}




\section{Introdução}

Estudos epidemiológicos têm utilizado questionários para obter o autorrelato acerca da demanda física no trabalho, pois além de ser uma técnica que permite investigar um grande número de trabalhadores, possibilita a inclusão de itens relativos a muitos aspectos teóricos de interesse e apresenta baixo custo $1,2,3,4$.

A mensuração da exposição à demanda física no trabalho em estudos epidemiológicos, em passado recente e até em dias mais atuais, não raras vezes, é feita baseada no título das ocupações, mas a correlação com a real exposição pode ser limitada com o uso desta alternativa 4,5,6. A insuficiência dessa medida baseada em títulos da ocupação tem conduzido aos estudos que buscam avaliar a validade e a confiabilidade da medida da demanda física ocupacional, por meio de questões que incorporem as diferentes modalidades de demanda física sobre o corpo no trabalho. Essa busca acompanha o crescimento dos estudos epidemiológicos e as novas abordagens acerca dos distúrbios musculoesqueléticos 7 . Como possível preditora desses agravos, a demanda física no trabalho precisa ser medida por meio de perguntas e escalas de resposta que confiram validade aos estudos epidemiológicos sobre sua associação com os distúrbios musculoesqueléticos.

Burdorf \& van der Beek 2 chamam a atenção para a complexidade da avaliação da exposição à demanda física no trabalho. Ressaltam muitas razões para essa complexidade, assinalando que a exposição na epidemiologia musculoesquelética não pode ser determinada por um único parâmetro, como é mais usual em outras áreas da epidemiologia ocupacional, quando a exposição se refere a um único agente ou fator do ambiente externo. Assim, cabe considerar que a demanda física no trabalho implica um construto com mais de uma dimensão. Incorpora posturas gerais, em pé, andando, agachado, adotadas com maior ou menor duração em situações de trabalho; posturas de segmentos corporais, como tronco, braços; movimentos repetitivos e manuseio de carga, que implica força. Essas são dimensões da demanda física nos cenários ocupacionais 1.

Apesar dessas diferentes dimensões que constituem a demanda física, e que, portanto, a distingue das demais demandas ocupacionais caracterizadas como um único agente de risco, estudos de validade de construto de demanda física não foram identificados em revisão sistemática realizada em 2005 1. A despeito de não existir um padrão-ouro para medida de demanda física no trabalho 8 , na referida revisão, todos os estudos identificados avaliaram validade de critério, investigando o desempenho do autorrelato de trabalhadores sobre demanda física no trabalho, por meio de questões específicas, comparando este autorrelato com outras técnicas (observacionais ou de medida direta). Muito recentemente, um estudo de validade de uma escala de demanda física voltada para extremidades superiores, embora também tenha avaliado validade de critério, já incluiu a validade estrutural desta escala, contribuindo com a ideia da demanda física como construto 8 .

Alguns autores 1 veem o emprego de avaliações interdisciplinares, incorporando técnicas de observação dos locais de trabalho e autorrelato de trabalhadores por meio de questionários, como a abordagem mais adequada e completa para a apreensão da demanda física no trabalho, no entanto, estas avaliações não se viabilizam no curso de estudos extensivos, de base populacional. Assim, avaliar o desempenho do autorrelato da demanda física, por meio do uso de questionário, considerando-se a dimensionalidade desta demanda ocupacional, pode contribuir com a epidemiologia musculoesquelética.

O autorrelato de trabalhadores pode ser capaz de descrever as particularidades da demanda física do trabalho, expressando a informação individual que incorpora toda a experiência de trabalho, com exigências variáveis, ao longo da jornada diária ou implicando variabilidade sazonal, o que é especialmente relevante nas ocupações mais dinâmicas. Ao contrário, as demais técnicas, observacionais e de medida direta, vão se restringir ao período amostrado, habitualmente com registros de curta duração 1,4.

Dentre os estudos que testaram validade de critério e confiabilidade de questões que medem demanda física no trabalho, que descrevemos em outra publicação 1, assinala-se aqui o desempenho de sete questões autoadministradas (The Stockholm Public Health Questionnaire) sobre movimentos repetitivos, posturas gerais do corpo, posturas anômalas específicas e manuseio de carga 9, e destacase também um instrumento finlandês que avaliou dez questões autoadministradas sobre postura geral do corpo, do tronco e do pescoço, dos braços acima dos ombros, manuseio de carga e movimentos repetitivos 10 . Não foram identificados estudos brasileiros que apresentassem validade e confiabilida- 
de de questionário sobre demanda física no trabalho, nem questionários estrangeiros validados com a população brasileira.

Este estudo teve por objetivo explorar a dimensionalidade, consistência interna e confiabilidade teste-reteste de um novo conjunto de itens desenvolvido para medir demanda física no trabalho.

\section{Métodos}

Estudo de corte transversal foi conduzido por meio de dois inquéritos em locais de trabalho, com a participação de 1.070 trabalhadores (228 mulheres e 842 homens) e 97\% de taxa de resposta. De duas empresas da indústria de calçados no interior da Bahia, Brasil, a amostra $(n=446)$ foi composta por operadores de montagem e acabamento na fabricação de calçados, operadores de máquinas, pessoal de manutenção e administração; da empresa de limpeza urbana, em Salvador, participaram trabalhadores $(n=624)$ inseridos nas ocupações de coletores de lixo, agentes de operações de limpeza especial, motoristas de caminhões e trabalhadores de manutenção. Todos os participantes estavam empregados quando o estudo foi conduzido.

Em ambos os inquéritos, para coletar os dados, foi aplicado um questionário por equipe de entrevistadores treinados, composta por engenheiro de segurança, três fisioterapeutas, um ergonomista e quatro acadêmicos de fisioterapia. Os dados foram coletados em cada empresa participante, durante a jornada de trabalho, em lugar reservado, garantindo privacidade. A coleta de dados foi precedida por reuniões nos locais de trabalho, nas quais informava-se aos trabalhadores os objetivos do estudo e a instituição responsável pela pesquisa, Universidade Federal da Bahia (UFBA). Visava-se a assegurar o direito à informação de cada sujeito da pesquisa, incrementar a adesão dos participantes e reduzir vieses de informação, característicos da pesquisa no mundo do trabalho.

\section{Desenvolvimento dos itens}

Utilizaram-se itens sobre postura geral do corpo, posturas de segmento corporal, repetitividade e manuseio de carga, obtidos por meio de questionário aplicado por entrevistador. Os itens foram inicialmente elaborados para compor o instrumento da pesquisa intitulada Distúrbios Musculoesqueléticos em Trabalhadores da Indústria 11.

A elaboração dos itens foi inspirada, entre outras fontes, em um questionário do Instituto Nacional de Pesquisa e Seguridade Francês 12, que reuniu instrumentos prévios. Desse questionário constam questões sobre "trabalho sentado", "força muscular requerida", "necessidade de movimentos precisos e muito finos" e a escala de resposta é do tipo visual analógico, com qualificadores verbais nas extremidades, sem numeração, e uma questão sobre "pressão exercida sobre o objeto de trabalho", com escala de resposta qualitativa e ordinal.

Dos dez itens do presente estudo, seis são relativos a posturas de trabalho, um sobre movimentos repetitivos com as mãos e três referentes a manuseio de carga (Figura 1). Apresentam escala de resposta do tipo Likert (escala ordinal), com seis pontos numéricos, de 0 a 5 . Nove dos dez itens apresentam escala que mensura duração, com qualificadores verbais nas extremidades $(0=$ jamais, $5=0$ tempo todo) e um, escala de intensidade ( $0=$ muito fraca, $5=$ muito forte). Os dez itens e suas escalas ora testados não são encontrados no instrumento francês referido ${ }^{12}$, que é mencionado apenas como fonte inspiradora.

Os itens foram inicialmente aplicados em uma população de trabalhadores da indústria de plástico, na Região Metropolitana de Salvador, em um projeto epidemiológico que investigava os fatores associados aos distúrbios muscuesqueléticos nesta população 13,14. Nos estudos desse projeto, conduziram-se análises sobre a associação da demanda física com os distúrbios musculoesqueléticos, encontrando-se associações positivas, consistentes e compatíveis com a teoria biomecânica entre exposição à demanda física e distúrbios musculoesqueléticos em pescoço, ombro, região dorsal e lombar. Os resultados empíricos 7,13,14,15,16,17 com os itens ora testados permitiram a superação da mensuração da demanda física por meio de título de ocupação, e geraram a pergunta de estudo sobre a dimensionalidade deste conjunto de itens que mede a demanda física no trabalho. 


\section{Figura 1}

Questionário de demanda física no trabalho.

Posturas:

Você trabalha: Jamais

O tempo todo

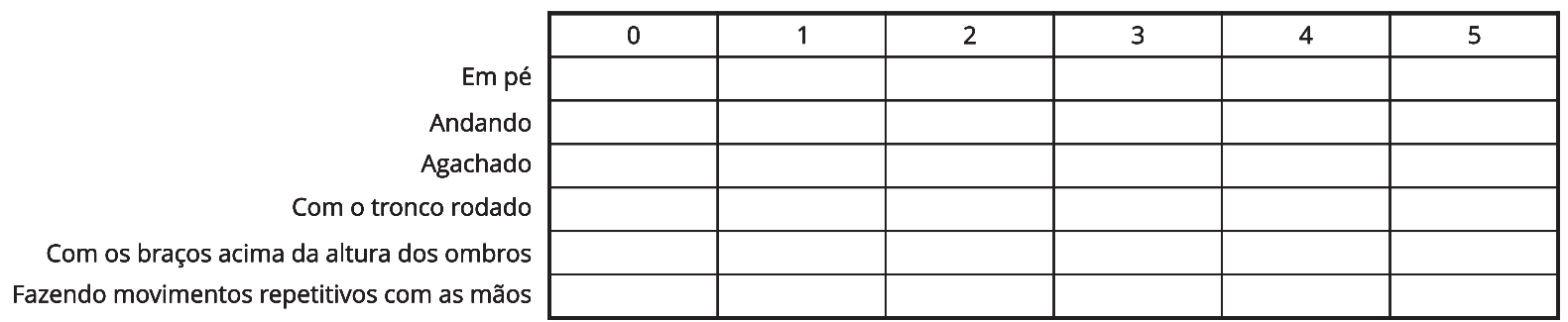

Pressão física com as mãos sobre objeto de trabalho:

Muito fraca
\begin{tabular}{|c|c|c|c|c|c|}
\hline 0 & 1 & 2 & 3 & 4 & 5 \\
\hline
\end{tabular}

Manuseio de carga:

Jamais
\begin{tabular}{|l|c|c|c|c|c|c|}
\hline & 0 & 1 & 2 & 3 & 4 & 5 \\
\cline { 2 - 7 } \\
\cline { 2 - 7 } Levantar \\
Puxar & & & & & & \\
\cline { 2 - 7 } & & & & & & \\
\cline { 2 - 7 }
\end{tabular}

\section{Etapas de análise}

1) Realizou-se análise fatorial exploratória e da consistência interna de dez itens de demanda física utilizados em duas populações distintas: (i) trabalhadores da indústria de calçados na Bahia, em 2012; (ii) trabalhadores de limpeza urbana, em Salvador, Bahia, em 2010.

Para investigar a dimensionalidade do construto demanda física no trabalho, utilizou-se a análise fatorial. Essa técnica permite descrever a variabilidade entre os itens originais, em termos de um número menor de variáveis, as dimensões comuns, que estão relacionadas com os itens por meio de um modelo linear 18. O método de estimação utilizado foi o Weighted Least Squares Mean and Variance Adjusted (WLSMV), mais apropriado para dados ordinais, conforme assinalam Barendse et al. 19. Como se trata da primeira investigação sobre a dimensionalidade desses itens que medem demanda física no trabalho e como não há uma hipótese sobre a quantidade de dimensões que devem fazer parte do modelo linear nem do seu significado, optou-se por utilizar a Análise Fatorial Exploratória. A rotação oblíqua Promax foi aplicada para a melhor interpretação dos valores. Ao considerar a estrutura da escala Likert, foram utilizadas matrizes de correlação policóricas. Para a análise da estrutura dimensional dos itens, obedeceu-se ao critério de Kaiser do autovalor (eingenvalue) maior que 1. A técnica de scree plot foi usada para a confirmação da quantidade de dimensões a serem extraídas. Os itens que apresentaram carga superior a 0,30 foram retidos para a composição das dimensões 20. Para essa etapa, foi utilizado o software Mplus 7.1 (https://www.statmodel.com/).

A qualidade do ajuste do modelo foi avaliada por três índices. O índice Root Mean Square Error of Aproximation (RMSEA), Comparative Fit Index (CFI) e Tucker-Lewis Index (TLI). RMSEA é uma medida da média da variância e da covariância residual. Valores de RMSEA menores ou iguais a 0,08 indicam um bom ajuste. Índices de ajuste comparativo, também conhecidos como índices de ajuste incremen- 
tais, avaliam o ajuste de um modelo proposto em relação a um modelo mais restrito. Ambos variam entre 0 e 1, valores maiores que 0,9 são indicativos de um bom ajuste 20 .

A consistência interna foi avaliada comparando-se as estimativas do alfa de Cronbach para as dimensões compostas pelos itens. Para o cálculo do alfa de Cronbach, foi usado o SPSS 15.0 (https:// www.ibm.com/) e adotados, como parâmetros de referência de desempenho satisfatório, valores de $0,65-0,9021$.

2) O estudo de confiabilidade teste-reteste utilizou uma subamostra de 121 trabalhadores selecionados aleatoriamente de uma das empresas de calçados. O tamanho da amostra foi calculado considerando-se o intervalo de 95\% de confiança (IC95\%), erro de 5\% e confiabilidade teste-reteste esperada de 0,70 , de acordo com Streiner \& Norman 22. Para este estudo, contou-se com a mesma equipe treinada, sendo que a segunda aplicação foi realizada por uma terceira fisioterapeuta, nas mesmas condições da primeira aplicação, ou seja, nos locais de trabalho, durante a jornada, assegurando privacidade.

Utilizando-se os mesmos dez itens já descritos, foi realizada a análise de confiabilidade com a utilização do índice kappa de ponderação quadrática, comparando-se os autorrelatos dos trabalhadores sobre demanda física obtidos na primeira fase (teste) aos da segunda fase (reteste). Conforme Streiner \& Norman 22, em casos de utilização de escalas Likert, as quais pressupõem valores iniciando em zero, que indicam a ausência de um atributo, passando por valores mais baixos até os mais altos, indicando presença crescente de um atributo, o uso do índice kappa ponderado é mais indicado que o kappa simples por resolver as discrepâncias entre um valor que considera o atributo inexistente e outro que o considera presente, embora em graus variados.

Para a interpretação do índice kappa (K) foi utilizada a classificação proposta por Landis \& Koch 23. Com o intuito de padronizar os dados da força de concordância pelo índice kappa, estes autores propuseram a seguinte classificação: $K<0,00$ - pobre; $K=0,00-0,20$ - fraco; $K=0,21-0,40$ - regular; $\mathrm{K}=$ 0,41-0,60 - moderado; $\mathrm{K}=0,61-0,80$ - bom; $\mathrm{K}=0,81-1,00$ - excelente.

A confiabilidade dos itens foi descrita para toda a amostra e de acordo com sexo, escolaridade e idade. Para a escolaridade, utilizaram-se os estratos: < 2o grau e $\geq 2$ o grau, e para idade, $\leq 28$ anos ou > 28 anos (mediana). A análise da significância estatística do kappa foi realizada observando se havia sobreposição entre os IC95\% para os estratos dessas variáveis.

A análise dos dados foi realizada usando-se os programas estatísticos software $\mathrm{R}$ versão 3.0.3 (http://www.r-project.org) e Stata 12 (https://www.stata.com).

\section{Ética em pesquisa}

Os projetos de pesquisa, na indústria de calçados e na limpeza urbana, foram aprovados, respectivamente, pelo Comitê de Ética e Pesquisa da Escola de Enfermagem da UFBA, sob o no 485004, e pelo Comitê de Ética em Pesquisa do Hospital São Rafael, protocolo no 48/09. Todos os participantes assinaram o Termo de Consentimento Livre e Esclarecido (TCLE), antes de serem entrevistados.

\section{Resultados}

\section{Sobre a dimensionalidade dos itens}

Foram estudados 1.070 trabalhadores. Na limpeza urbana foram estudados 624 trabalhadores, todos do sexo masculino, com mediana de idade de 33 anos. Desses, 37\% tinham concluído o Ensino Médio. Cerca de 55\% se autoclassificaram negros e 34\% pardos. Na indústria de calçados, foram estudadas 228 mulheres e 218 homens, cuja mediana de idade foi 29 anos. Desses 446 trabalhadores, $78 \%$ tinham concluído o ensino médio completo, 32,5\% se autoclassificaram negros e 51,3\% pardos. Foram estudados no total 1.070 trabalhadores (taxa de resposta de 97\%).

A análise fatorial exploratória capturou os dez itens de demanda física em duas dimensões relativas ao trabalho na limpeza urbana e em três no trabalho industrial de fabricação de calçados (Figura 2).

As dimensões identificadas neste estudo podem ser descritas como: (1) trabalho dinâmico com posturas anômalas; (2) trabalho que implica manuseio de material; e (3) trabalho com exigência de repetitividade com as mãos. 
Figura 2

Scree plot da análise fatorial dos itens sobre demanda física no trabalho, em duas populações de trabalhadores.

2a) Análise fatorial - limpeza urbana

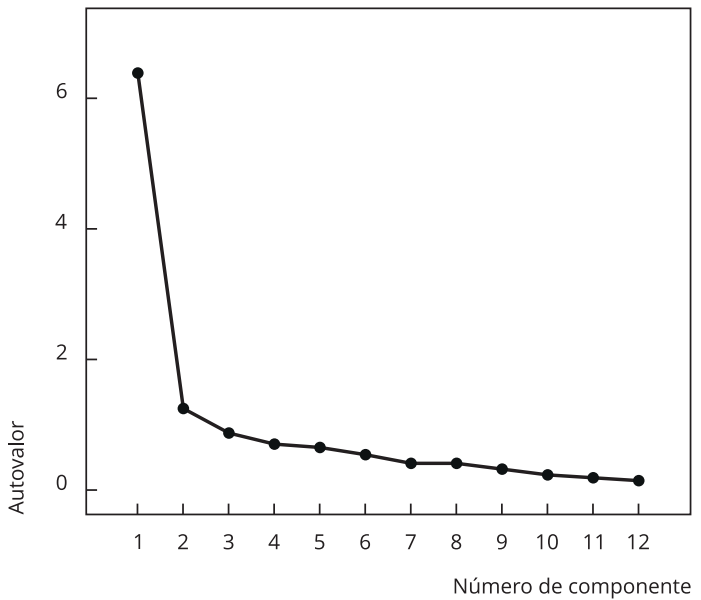

2b) Análise fatorial - indústria de calçados

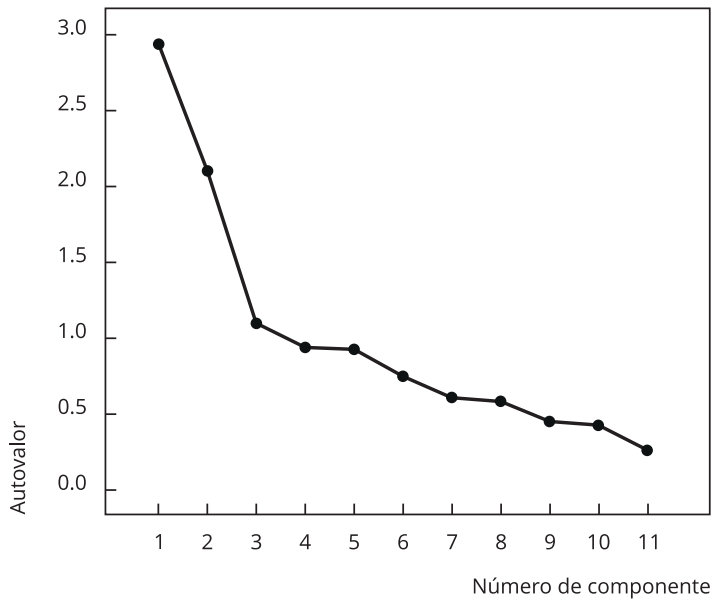

A composição das dimensões, em ordem decrescente das cargas apresentadas por cada item, foi a seguinte no trabalho na limpeza urbana: Dimensão 1 (eigenvalue inicial = 5,856; 58,55\%) caracterizou o trabalho em pé $(0,96)$, adotando também postura agachada $(0,80)$, convocando postura não neutra do tronco e braços, isto é, tronco rodado $(0,73)$, com braços acima do nível dos ombros $(0,73)$, andando $(0,61)$, além de implicar movimentos repetitivos com as mãos $(0,44)$; Dimensão 2 (eigenvalue = 1,174; $11,74 \%)$ caracterizou o manuseio de materiais, incluindo puxar $(0,98)$, empurrar $(0,92)$ e levantar cargas $(0,89)$, fazendo pressão com as mãos sobre o objeto de trabalho $(0,33)$. O eigenvalue acumulado pelas duas dimensões extraídas foi de 70,29\%, indicando uma boa explicação do conjunto de itens. Já a composição das três dimensões, também em ordem decrescente das cargas apresentadas por cada item no trabalho industrial de fabricação de calçados, foi: Dimensão 1 (eigenvalue inicial = 3,372; $33,71 \%)$ caracterizou o trabalho agachado $(0,83)$, andando $(0,61)$, em pé $(0,49)$, com braços acima do nível dos ombros (0,43); Dimensão 2 (eigenvalue $=2,265 ; 22,65 \%$ ) caracterizou o manuseio de cargas, incluindo puxar $(0,94)$, empurrar $(0,89)$ e levantar $(0,79)$ materiais; Dimensão 3 (eigenvalue = 1,147; $11,47 \%)$ caracterizou o trabalho que requer movimentos repetitivos com as mãos $(0,89)$, tronco rodado $(0,60)$, exercendo pressão física sobre o objeto de trabalho $(0,39)$. O eigenvalue acumulado pelas três dimensões extraídas foi de 67,83\%, indicando, também neste caso, uma boa explicação do conjunto de itens. A qualidade do ajuste dos dois modelos foi adequada, segundo os três índices utilizados (RMSEA, CFI, TLI) (Tabela 1).

\section{Consistência interna}

A consistência interna, medida pelo alfa de Cronbach, revelou alta correlação entre as questões que compõem as duas dimensões para exposição à demanda física na limpeza urbana $(\alpha=0,84)$. Os itens que compõem a segunda dimensão para o trabalho industrial em calçados apresentam-se da mesma forma altamente correlacionados, tratando-se de manuseio de material, que incorpora puxar, empurrar e levantar materiais $(\alpha=0,84)$. No entanto, a consistência interna foi mais baixa para as dimensões que caracterizam o trabalho dinâmico (Dimensão 1) e aquela com posturas anômalas e repetitividade (Dimensão 3), nesse contexto industrial (Tabela 1). 


\section{Tabela 1}

Estrutura fatorial e consistência interna de itens sobre demanda física no trabalho, em duas populações de trabalhadores.

\begin{tabular}{|c|c|c|c|c|c|}
\hline \multirow[t]{2}{*}{ Itens } & \multicolumn{2}{|c|}{$\begin{array}{l}\text { Limpeza urbana } \\
\qquad(n=624)\end{array}$} & \multicolumn{3}{|c|}{$\begin{array}{l}\text { Indústria de calçados } \\
\qquad(n=446)\end{array}$} \\
\hline & D1 & D2 & D1 & D2 & D3 \\
\hline Em pé & 0,96 & & 0,49 & & \\
\hline Andando & 0,61 & & 0,61 & & \\
\hline Agachado & 0,80 & & 0,83 & & \\
\hline Tronco rodado & 0,73 & & & & 0,60 \\
\hline Braços acima dos ombros & 0,73 & & 0,43 & & \\
\hline Movimentos repetitivos & 0,44 & & & & 0,89 \\
\hline Levantar carga & & 0,89 & & 0,79 & \\
\hline Empurrar & & 0,92 & & 0,89 & \\
\hline Puxar carga & & 0,98 & & 0,94 & \\
\hline Pressão física com as mãos & & 0,33 & & & 0,39 \\
\hline Eigenvalues & 5,856 & 1,174 & 3,372 & 2,265 & 1,147 \\
\hline Eigenvalues (\% acumulado) & 58,55 & 70,29 & 33,71 & 56,36 & 67,83 \\
\hline RMSEA (IC90\%) & \multicolumn{2}{|c|}{$0,045(0,027-0,064)$} & \multicolumn{3}{|c|}{$0,021(0,000-0,049)$} \\
\hline $\mathrm{CFI}$ & \multicolumn{2}{|c|}{0,999} & \multicolumn{3}{|c|}{0,999} \\
\hline TLI & \multicolumn{2}{|c|}{0,997} & \multicolumn{3}{|c|}{0,997} \\
\hline Alfa de Cronbach & 0,84 & 0,84 & 0,59 & 0,84 & 0,55 \\
\hline
\end{tabular}

CFI: Comparative Fit Index; RMSEA: Root Mean Square Error of Aproximation; TLI: Tucker-Lewis Index.

Nota: D1 = Dimensão 1 - trabalho dinâmico com posturas anômalas; D2 = Dimensão 2 - trabalho que implica manuseio de material; D3 = Dimensão 3 - trabalho com exigência de repetitividade com as mãos.

\section{Confiabilidade teste-reteste}

O teste-reteste foi realizado no tempo médio de 33 dias (21 a 45 dias).

$\mathrm{Na}$ Tabela 2, observa-se para o item postura em pé confiabilidade excelente, boa para a postura andando e moderada para trabalho agachado. A confiabilidade foi moderada para pressão com as mãos sobre objeto de trabalho e manuseio de cargas (levantar e empurrar). Além disso, em postura com segmento corporal, a rotação de tronco apresentou kappa moderado, e a postura de braços acima do nível dos ombros, bem como movimentos repetitivos, confiabilidade apenas regular.

Ao se analisar a confiabilidade do autorrelato de demanda física, de acordo com o sexo e a escolaridade, é possível afirmar que não há diferença estatisticamente significante entre os estratos dos itens; apenas o item tronco rodado apresentou diferença estatisticamente significante de acordo com a idade, com o autorrelato mais confiável entre aqueles com menos de 28 anos de idade (Tabela 3).

\section{Discussão}

Os resultados apresentados sugerem dimensionalidade dos itens de demanda física no trabalho, em especial, para medida da exposição desta demanda em estudos sobre distúrbios musculoesqueléticos. A avaliação da exposição ocupacional na epidemiologia musculoesquelética ainda enseja debate acerca da validade de técnicas de mensuração, contudo, o papel central do autorrelato acerca da demanda física, obtido por meio de questionário, tem sido reconhecido no contexto de estudos extensivos com populações de trabalhadores. Assim, avaliar o desempenho do autorrelato da demanda física, por meio do uso de questionário, considerando-se a dimensionalidade desta demanda ocupacional, pode contribuir com a literatura científica em epidemiologia musculoesquelética. Ademais, os distúrbios 
Tabela 2

Resultados da confiabilidade do autorrelato de demandas físicas em trabalhadores da indústria de calçados.

\begin{tabular}{lcccc}
\hline Questões & $\begin{array}{c}\text { Concordância } \\
\text { observada (\%) }\end{array}$ & $\begin{array}{c}\text { Concordância } \\
\text { esperada (\%) }\end{array}$ & $\begin{array}{c}\text { Kappa } \\
\text { ponderado }\end{array}$ & $\begin{array}{c}\text { Classificação } \\
\text { kappa * }\end{array}$ \\
\hline Em pé & 95,6 & 71,1 & 0,84 & Excelente \\
Andando & 91,2 & 72,9 & 0,67 & Bom \\
Agachado & 94,3 & 87,7 & 0,53 & Moderado \\
Tronco rodado & 84,6 & 70,9 & 0,46 & Regular \\
Braços acima dos ombros & 93,6 & 90,6 & 0,31 & Regular \\
Movimentos repetitivos com as mãos & 89,2 & 82,0 & 0,39 & Moderado \\
Levantar cargas & 89,6 & 75,4 & 0,57 & Regular \\
Puxar cargas & 86,2 & 78,7 & 0,35 & Moderado \\
Empurrar cargas & 89,0 & 80,2 & 0,44 & Moderado \\
Pressão física com as mãos sobre objeto & 89,9 & 81,2 & 0,46 & \\
de trabalho & & & & \\
\hline
\end{tabular}

* Classificação segundo Landis \& Koch 23: ruim (<0,00); pobre $(0,00-0,20)$; regular $(0,21-0,40)$; moderada $(0,41-0,60)$; boa $(0,61-0,80)$; excelente $(0,81-1,00)$

musculoesqueléticos ocupam lugar de destaque na agenda dos agravos relacionados ao trabalho, e disponibilizar um novo instrumento de medida da exposição preditora destes agravos pode ser uma contribuição relevante para a comunidade.

\section{Dimensionalidade dos itens de demanda física no trabalho}

As dimensões nas quais os dez itens que descrevem demanda física no trabalho foram capturados na análise fatorial exploratória são compatíveis com a natureza da exposição à demanda física, na limpeza urbana e na indústria de calçados. Os resultados revelam as especificidades de cada grupo ocupacional, o que pode sugerir dimensionalidade dos itens de demanda física que, em geral, mostraram boa consistência interna.

A população estudada na limpeza urbana, para a qual foram identificadas duas dimensões da demanda física, é composta por coletores de lixo, em sua maioria, cujas tarefas implicam manuseio de material na coleta do lixo em tonéis, nas vias públicas e no deslocamento desta carga para acondicioná-la em caminhões. Essa é a centralidade da exposição nessa população e sua descrição pode ser obtida nos resultados da análise ergonômica do trabalho por Camada et al. 24.

$\mathrm{Na}$ indústria de calçados, as três dimensões, por certo, refletem a maior variabilidade de exposição à demanda física no trabalho nesta atividade industrial. Nesse cenário ocupacional, a demanda física varia consideravelmente das linhas de fabricação e montagem de calçados, passando pela costura dos acessórios de cada modelo, à operação de máquinas e tarefas de manutenção 25,26.

A primeira dimensão, trabalho dinâmico com posturas anômalas, constituída pelos itens trabalho em pé, andando, agachado, com braços elevados acima da altura dos ombros e tronco rodado, expressa em larga medida as tarefas na limpeza urbana realizadas pelos coletores, motoristas, agentes especiais e pessoal de manutenção, que se caracterizam pelo trabalho executado de forma dinâmica, habitualmente exigindo postura ortostática e em movimento, posturas não neutras do tronco e membros superiores, para o alcance de equipamentos ou operação de ferramentas. As altas cargas dos itens dessa dimensão na limpeza urbana variam de 0,61-0,96, com carga menos relevante apenas para o item movimentos repetitivos com as mãos $(0,44)$. Na indústria de calçados, atividades de manutenção e a pré-fabricação de calçados (modelagem e corte) podem exigir o trabalho andando ou agachado, e a atividade de fabricação de calçados propriamente dita (montagem e acabamento de calçados) implica, principalmente, o trabalho em pé mantido e postura não neutra dos braços - elevados acima do nível dos ombros. Nesse cenário da indústria, as cargas dos itens que compuseram essa dimensão variaram de $0,43-0,83$. 


\section{Tabela 3}

Confiabilidade (kappa) e intervalo de 95\% de confiança (IC95\%) para o autorrelato de demanda física, de acordo com sexo, escolaridade e idade.

\begin{tabular}{|c|c|c|}
\hline \multirow[t]{2}{*}{ Questões } & \multicolumn{2}{|c|}{ Sexo (IC95\%) } \\
\hline & Masculino [n = 55] & Feminino [n = 66] \\
\hline Em pé & $0,915(0,818 ; 0,974)$ & $0,806(0,642 ; 0,920)$ \\
\hline Andando & $0,707(0,501 ; 0,863)$ & $0,584(0,362 ; 0,741)$ \\
\hline Agachado & $0,573(0,337 ; 0,738)$ & $0,480(0,172 ; 0,713)$ \\
\hline Tronco rodado & $0,461(0,188 ; 0,694)$ & $0,475(0,254 ; 0,664)$ \\
\hline Braços acima dos ombros & $0,455(0,108 ; 0,689)$ & $0,222(-0,027 ; 0,575)$ \\
\hline Movimentos repetitivos com as mãos & $0,389(0,135 ; 0,600)$ & $0,345(0,121 ; 0,603)$ \\
\hline Levantar cargas & $0,512(0,282 ; 0,719)$ & $0,611(0,393 ; 0,798)$ \\
\hline Puxar cargas & $0,483(0,195 ; 0,700)$ & $0,177(-0,091 ; 0,461)$ \\
\hline Empurrar cargas & $0,519(0,292 ; 0,719)$ & $0,329(0,065 ; 0,580)$ \\
\hline \multirow[t]{3}{*}{ Pressão física com as mãos } & $0,486(0,262 ; 0,659)$ & $0,443(0,185 ; 0,652)$ \\
\hline & \multicolumn{2}{|c|}{ Escolaridade } \\
\hline & $<2^{\circ}$ grau $[\mathrm{n}=37]$ & $\geq 2^{\circ}$ grau $[n=84]$ \\
\hline Em pé & $0,930(0,821 ; 0,987)$ & $0,815(0,676 ; 0,911)$ \\
\hline Andando & $0,784(0,576 ; 0,921)$ & $0,611(0,432 ; 0,764)$ \\
\hline Agachado & $0,381(0,111 ; 0,643)$ & $0,603(0,403 ; 0,736)$ \\
\hline Tronco rodado & $0,242(-0,078 ; 0,546)$ & $0,533(0,343 ; 0,704)$ \\
\hline Braços acima dos ombros & $0,310(0,007 ; 0,635)$ & $0,326(0,056 ; 0,626)$ \\
\hline Movimentos repetitivos com as mãos & $0,166(0,003 ; 0,438)$ & $0,522(0,319 ; 0,692)$ \\
\hline Levantar cargas & $0,461(0,173 ; 0,707)$ & $0,622(0,431 ; 0,784)$ \\
\hline Puxar cargas & $0,368(0,134 ; 0,647)$ & $0,339(0,107 ; 0,539)$ \\
\hline Empurrar cargas & $0,712(0,484 ; 0,876)$ & $0,301(0,093 ; 0,520)$ \\
\hline \multirow[t]{3}{*}{ Pressão física com as mãos } & $0,544(0,310 ; 0,746)$ & $0,405(0,179 ; 0,594)$ \\
\hline & \multicolumn{2}{|c|}{ Idade } \\
\hline & $\leq 28$ anos $[n=75]$ & $>28$ anos $[n=46]$ \\
\hline Em pé & $0,847(0,709 ; 0,940)$ & $0,849(0,651 ; 0,949)$ \\
\hline Andando & $0,699(0,496 ; 0,834)$ & $0,643(0,390 ; 0,803)$ \\
\hline Agachado & $0,512(0,238 ; 0,699)$ & $0,555(0,364 ; 0,734)$ \\
\hline Tronco rodado & $0,675(0,491 ; 0,827)$ & $0,211(0,078 ; 0,473)$ \\
\hline Braços acima dos ombros & $0,155(-0,089 ; 0,486)$ & $0,487(0,208 ; 0,697)$ \\
\hline Movimentos repetitivos com as mãos & $0,364(0,137 ; 0,611)$ & $0,445(0,213 ; 0,680)$ \\
\hline Levantar cargas & $0,576(0,357 ; 0,749)$ & $0,564(0,333 ; 0,767)$ \\
\hline Puxar cargas & $0,450(0,179 ; 0,676)$ & $0,241(-0,004 ; 0,514)$ \\
\hline Empurrar cargas & $0,430(0,182 ; 0,688)$ & $0,437(0,177 ; 0,688)$ \\
\hline Pressão física com as mãos & $0,461(0,205 ; 0,643)$ & $0,457(0,206 ; 0,679)$ \\
\hline
\end{tabular}

Na segunda dimensão, trabalho que implica manuseio de material, os itens puxar, empurrar e levantar cargas caracterizam tarefas que acionam todo o corpo do trabalhador. Na limpeza urbana, esses itens apresentam altas cargas, que variam de 0,89-0,98, e esta dimensão inclui o item exercer pressão física com as mãos sobre objeto de trabalho, que nesta população pode expressar o uso das mãos em pega ou garra de equipamentos e ferramentas de trabalho. Esse item apresentou a mais baixa carga (0,33). Também no cenário ocupacional da indústria de calçados, os itens levantar, empurrar e puxar material apresentam altas cargas $(0,79-0,94)$. 
$\mathrm{Na}$ limpeza urbana, ambas as dimensões identificadas no presente estudo apresentam forte consistência interna dos itens $(0,84)$, ratificando a quase indissociabilidade, por um lado, nas tarefas de manuseio de material, do levantamento e dos atos de puxar e empurrar cargas, e nas tarefas dinâmicas, da posição em pé, andando, agachado e das posturas não neutras do tronco e dos membros superiores. Observou-se que também no contexto da indústria de calçados a segunda dimensão, trabalho que implica manuseio de material, apresentou uma boa consistência interna dos seus itens $(0,84)$, reforçando a forte correlação entre levantar, puxar e empurrar cargas, no entanto, neste contexto da indústria, a consistência interna foi pobre para a primeira dimensão.

A terceira dimensão que trata do trabalho com exigência de repetitividade com as mãos, identificada no trabalho da indústria de calçados, traz, preponderantemente, considerando-se a carga apresentada $(0,89)$, o item repetitividade com as mãos, mas a postura não neutra do tronco $(0,60)$ e a pressão física com as mãos sobre o objeto de trabalho $(0,39)$ também compõem esta dimensão na indústria de calçados. Essa dimensão apresenta a mais baixa consistência interna dos itens. No cenário de exposição ocupacional na indústria de calçados, há uma maior variabilidade de tarefas; ademais, diferentes combinações de uso do corpo são requeridas, as quais podem implicar diferentes padrões de exigência física durante a jornada de trabalho com algumas tarefas realizadas por períodos mais curtos. É possível discutir se essas peculiaridades poderiam resultar em maior dificuldade para os trabalhadores fornecerem uma resposta mais confiável, haja vista que a variabilidade intrassujeito pode resultar em alfa de Cronbach subestimado. A repetitividade de movimentos com as mãos na fabricação de calçados é um achado central em muitas etapas do processo produtivo 26,27,28,29. Esse item carrega fortemente nessa terceira dimensão, que não foi identificada na limpeza urbana, na qual a repetitividade de movimentos com as mãos carregou na Dimensão 1. No conjunto das tarefas da indústria de calçados, a repetitividade no uso das mãos assumiu lugar de destaque dada a sua forte presença desde a pré-fabricação de calçados (modelagem e corte), fabricação de calçados propriamente dita (pesponto, montagem e acabamento) e, especialmente, na fabricação de acessórios com operações de costura 25,26.

\section{Confiabilidade teste-reteste}

A confiabilidade dos itens vai ao encontro da literatura, revelando, por um lado, excelente e boa confiabilidade para postura em pé e andando, respectivamente, moderada confiabilidade para postura agachada, manuseio de carga e postura de tronco rodado e, por outro lado, confiabilidade apenas regular para movimentos repetitivos. Itens envolvendo segmentos do corpo e repetitividade não têm reprodutibilidade tão boa como as posturas gerais 1. É importante ressaltar que a confiabilidade de um instrumento de medida é uma estimativa da sua aplicação em uma dada população e não deve ser considerada como indicador do instrumento em si mesmo.

Embora o trabalho agachado seja referido na literatura com resultados mais pobres de confiabilidade do que as demais posturas gerais, atribuídos ao fato desta postura de trabalho ocupar pouco tempo da jornada e por isto ser de difícil memorização e registro no autorrelato, neste estudo a confiabilidade deste item foi moderada, superando o achado de outras pesquisas ${ }^{1}$. Tronco rodado e pressão física com as mãos sobre o objeto de trabalho apresentaram confiabilidade moderada e foram consistentes com outros estudos 2,30,31,32,33,34. Os itens sobre manuseio de cargas registraram confiabilidade compatível com outros estudos, em especial, levantar e empurrar pesos, que apresentaram moderada confiabilidade 30,31,32,33,34.

Este estudo testou escala de resposta de seis pontos, medindo duração das demandas no trabalho, permitindo respostas menos rígidas, variando de "jamais" $(=0)$ a "o tempo todo" $(=5)$, fornecendo a ordem numérica e evitando escala mais extensa. Isso pode ter propiciado a obtenção de resultados de confiabilidade satisfatória para a maioria dos itens. Conforme Burdorf \& van der Beek 3 , as escalas que exigem níveis absolutos de exposição podem ser difíceis para o trabalhador, a exemplo da formulação "quanto tempo você trabalhou com as costas inclinadas para a frente mais de $45^{\circ}$ no turno passado?". Assim, questões que exigem menos detalhes podem ser mais facilmente respondidas e, portanto, de forma mais confiável e válida.

$\mathrm{Na}$ avaliação da confiabilidade, outro critério relevante diz respeito à independência entre as avaliações, ou seja, ao tempo entre o teste e reteste, que não deve ser muito curto por conta do viés 
de memória e nem muito longo para que não ocorram modificações nas condições de exposição que estão sob avaliação ${ }^{1}$. Neste estudo, o intervalo médio foi de 33 dias, compatível com o que a literatura propõe.

Stock et al. 1 expõem que os estudos devem ter adequado tamanho amostral e também variabilidade de exposição e número suficiente de indivíduos com alta, média e baixa exposição, haja vista que uma alta concordância esperada ao acaso, que pode ocorrer com a concentração de respostas em um único ponto da escala, pode resultar em valores baixos de kappa, apenas pelo efeito da baixa variabilidade de respostas 1,35,36. Embora os 121 indivíduos, participantes do teste-reteste, estivessem inseridos em ocupações leves e administrativas, ocupações de manutenção, pré-fabricação e fabricação de calçados, sendo contemplados, desta forma, todos os níveis de exposição, alguns itens apresentaram resposta concentrada em uma extremidade da escala, com possível efeito sobre o kappa, como ocorreu para o item braços acima da altura dos ombros, cuja confiabilidade foi apenas regular. Wiktorin et al. 33 apresentaram resultado próximo ao do presente estudo com confiabilidade moderada para esse item.

A confiabilidade para a postura tronco rodado foi moderada, assim como no estudo de Speklé et al. 37. Para movimentos repetitivos com as mãos o kappa foi regular, conforme também encontrado por boa parte dos autores que obtiveram de boa a excelente confiabilidade para questões sobre posturas gerais e piores desempenhos para o autorrelato de repetitividade 32,33,34.

Wiktorin et al. 33 relataram que o nível de educação pode influenciar no desempenho do autorrelato, entretanto, no presente estudo, não houve diferença entre as respostas fornecidas pelos indivíduos com maior ou menor grau de escolaridade. É possível que o saber-fazer adquirido ao longo do tempo de trabalho executando as tarefas influencie mais decisivamente para respostas mais confiáveis do que a educação formalmente adquirida, medida pelo grau de escolaridade. Quanto ao sexo, os dez itens avaliados neste estudo não foram discordantes entre homens e mulheres, muito embora outros autores 34 relatem que os homens parecem responder com mais confiabilidade que as mulheres. A idade não influenciou na confiabilidade do autorrelato, excetuando-se o item tronco rodado, que foi mais confiável entre os indivíduos com menos de 28 anos de idade. Não foram encontradas diferenças na variabilidade da exposição que explicassem os achados. Na literatura, não foram observadas diferenças para a confiabilidade do autorrelato de acordo com a idade 1,9,32,33.

\section{O autorrelato de demanda física e outras técnicas: limites e possibilidades}

Questionário, como recurso para a obtenção do autorrelato, constitui uma das técnicas possíveis para mensuração da demanda física no trabalho. E sobre as diferentes técnicas para esse fim, limites e vantagens têm sido apontados por autores que assinalam os desafios para essa medida 2,4,5,6,8.

Os questionários, frequentemente usados em estudos populacionais para medida de exposição e de desfecho, são criticados quanto à sua validade e confiabilidade para medida da demanda física no trabalho, no entanto, as técnicas para comparação com os questionários, utilizadas nos estudos de validade de critério, apresentam importantes limites 1,8 .

As técnicas observacionais para a identificação da demanda física no trabalho, em suas diferentes dimensões, são de mais alto custo que os questionários e limitadas para uso com grandes contingentes populacionais. Ademais, em trabalho dinâmico com tarefas que implicam grande variabilidade do uso do corpo, o uso de técnicas observacionais em uma amostra do tempo de trabalho pode não representar razoavelmente todo o espectro da exposição. $\mathrm{O}$ mesmo pode ser dito acerca das técnicas de medida direta (ou de instrumentação direta) 1,5.

Estudos sobre validade de critério do autorrelato de demanda física apresentam limites, pela ausência do verdadeiro padrão-ouro, conforme também constata um estudo publicado em 2017, que investigou validade de critério e validade estrutural da escala sobre demanda no trabalho para as extremidades superiores 8 .

Os questionários têm sido a técnica viável para abordagens extensivas, populacionais, portanto, a obtenção de medidas válidas, baseadas no autorrelato, permanece como tarefa posta aos pesquisadores e neste sentido é que se insere o presente estudo que avaliou a dimensionalidade de itens de demanda física no trabalho.

Para Streiner \& Norman 22, a validade estrutural é um processo contínuo, quase uma tarefa infindável para avaliar o desempenho de um instrumento em uma variedade de situações. Para esses 
autores, a pergunta central dos processos de validação deve ser se a hipótese faz sentido à luz do que o instrumento é desenhado para medir. A partir disso, neste estudo, a hipótese norteadora parece adequada: trabalhadores com altos escores para cada uma das três dimensões do construto demanda física poderão ter mais alto risco para os distúrbios musculoesqueléticos.

\section{Conclusões}

As dimensões identificadas neste estudo com base nos dez itens de demanda física podem ser descritas como trabalho dinâmico com posturas anômalas, trabalho que implica manuseio de material e trabalho com exigência de repetitividade com as mãos.

A confiabilidade por meio do teste-reteste revela, por um lado, de excelente a boa confiabilidade para postura geral de trabalho, moderada confiabilidade para manuseio de material, apresentando resultado apenas regular para movimentos repetitivos.

Os resultados revelam as especificidades de cada grupo ocupacional, o que pode sugerir dimensionalidade dos itens de demanda física, além de mostrarem confiabilidade satisfatória dos itens.

\section{Colaboradores}

R. C. P. Fernandes contribuiu na concepção, análise, interpretação dos dados e redação do artigo; responsável por todos os aspectos do trabalho na garantia da exatidão e integridade de qualquer parte da obra. L. P. Cunha participou da coleta, análise, interpretação dos dados e redação do artigo. V. M. C. Lima participou da análise dos dados e revisão crítica relevante do conteúdo intelectual. K. O. B. Santos participou da análise, interpretação dos dados e revisão crítica relevante do conteúdo intelectual. Todos os autores aprovaram a versão final do artigo, a ser publicada.

\section{Referências}

1. Stock SR, Fernandes R, Delisle A, Vézina N. Reproducibility and validity of workers' selfreports of physical work demands. Scand J Work Environ Health 2005; 31:409-37.

2. Burdorf A, van der Beek A. Exposure assessment strategies for work-related risk factors for musculoskeletal disorders. Scand J Work Environ Health 1999; 25 Suppl 4:25-30.

3. Burdorf A, van der Beek A. In musculoskeletal epidemiology are we asking the unanswerable in questionnaires on physical load? Scand J Work Environ Health 1999; 25:81-3.

4. Gardner BT, Lombardi DA, Dale AM, Franzblau A, Evanoff BA. Reliability of job-title based physical work exposures for the upper extremity: comparision to self-reported and observed exposure estimates. Occup Environ Med 2010; 67:538-47.

5. Winkel J, Mathiassen SE. Assessment of physical work load in epidemiologic studies: concepts, issues and operational considerations. Ergonomics 1994; 37:979-88.

6. Burdorf A, Rossignol M, Fathallah FA, Snook $\mathrm{SH}$, Herrick RF. Challenges in assessing risk factors in epidemiologic studies on back disorders. Am J Ind Med 1997; 32:142-52.

7. Fernandes RCP, Pataro SMS, Carvalho RB, Burdorf A. The concurrence of musculoskeletal pain and associated work-related factors: a cross sectional study. BMC Public Health 2016; 16:628.

8. Jacobs NW, Berduszek RJ, Dijkstra PU, van der Sluis CK. Validity and reliability of the upper extremity work demands scale. J Occup Rehabil 2017; 27:520-9. 
9. Leijon O, Wiktorin C, Harenstam A, Karlqvist L; Moa Research Group. Validity of a self-administered questionnaire for assessing physical workloads in a general population. J Occup Environ Med 2002; 8:724-35.

10. Viikari-Juntura E, Rauas S, Martikainen R, Kuosma E, Riihimäki H, Takala EP, et al. Validity of self-reported physical work load in epidemiologic studies on musculoskeletal disorders. Scand J Work Environ Health 1996; 22:251-9.

11. Fernandes RCP. Distúrbios musculoesqueléticos e trabalho industrial [Tese de Doutorado]. Salvador: Instituto de Saúde Coletiva, Universidade Federal da Bahia; 2004.

12. Cail F, Aptel M, Pichene A. Questionnaire d'evaluation du vécu du travail de salaries exposés à dês risques de trubles musculosquelettiques. Paris: Institut National de Recherche et de Sécurité; 1995. (Documents pour le Médecin Du Travail, 64).

13. Fernandes RCP, Carvalho FM, Assunção AA, Silvany Neto AA. Interactions between physical and psychosocial demands of work associated to low back pain. Rev Saúde Pública 2009; 43:326-34.

14. Fernandes RCP, Assunção AA, Silvany-Neto AM, Carvalho FM. Musculoskeletal disorders among workers in plastic manufaturing plants. Rev Bras Epidemiol 2010; 13:11-20.

15. Fonseca NR, Fernandes RCP. Factors related to musculoskeletal disorders in nursing workers. Rev Latinoam Enferm 2010; 18:1076-83.

16. Mascarenhas ALM, Fernandes RCP. Aptidão física e trabalho físico pesado: como interagem para a ocorrência de distúrbio musculoesquelético? Cad Saúde Pública 2014; 30:2187-98.

17. Pataro SMS, Fernandes RCP. Trabalho físico pesado e dor lombar: a realidade na limpeza urbana. Rev Bras Epidemiol 2014; 17:17-31.

18. Mingotti SA. Análise de dados através de métodos de estatística multivariada: uma abordagem aplicada. Belo Horizonte: Editora UFMG; 2005.

19. Barendse MT, Oort FJ, Timmerman ME. Using exploratory factor analysis to determine the dimensionality of discrete responses. Struct Equ Modeling 2015; 22:87-101.

20. Brown TA. Confirmatory factor analysis for applied research. New York: Guilford Press; 2006.

21. Nunnaly J. Psychometric theory. New York: McGraw-Hill; 1978.

22. Streiner DL, Norman GR. Health measurement scales: a practical guide to their development and use. 4th Ed. London: Oxford Medical Publications; 2008.

23. Landis JR, Koch GG. The measurement of observer agreement for categorical data. Biometrics 1977; 33:159-74.

24. Camada IM, Pataro SM, Fernandes RCP. Heavy physical work under time pressure: the garbage collection service-a case study. Work 2012; 41 Suppl 1:462-9.
25. Cunha LP. Autorrelato de trabalhadores sobre exposição às demandas físicas [Dissertação de Mestrado]. Salvador: Faculdade de Medicina da Bahia, Universidade Federal da Bahia; 2014.

26. Prazeres TJ, Navarro VL. Na costura do sapato, o desmanche das operárias: estudo das condições de trabalho e saúde das pespontadeiras da indústria de calçados de Franca, São Paulo, Brasil. Cad Saúde Pública 2011; 27:1930-8.

27. Carvalho RB, Lima VC, Fernandes RCP. Demandas psicológicas, baixo apoio social e repetitividade: fatores ocupacionais associados à dor musculoesquelética em trabalhadores da indústria de calçados. Rev Bras Saúde Ocup; no prelo.

28. Todd L, Puangthongthub ST, Mottus K, Mihlan G, Wing S. Health survey of workers exposed to mixed solvent and ergonomic hazards in footwear and equipment factory workers in Thailand. Ann Occup Hyg 2008; 52:195-205.

29. Roquelaure Y, Mariel J, Fanello S, Dano C, Penneau-Fontbonne D. Prevalence, incidence and risk factors of carpal tunnel syndrome in a large footwear factory. Int J Occup Med Environ Health 2001; 14:357-67.

30. Balogh I, Orback P, Winkel I, Nordander C, Ohlsson K, Ektor-Amdersen I. Questionnaire - based mechanical exposure indices for large population studies, reliability, internal consistence and predictive validity. Scand J Work Environ Health 2001; 27:41-8.

31. Halpern M, Hiebert R, Nordin M, Goldsheyder D, Crane M. The test-retest reliability of a new occupational risk factor questionnaire for outcome studies of low back pain. Appl Ergon 2001; 32:39-46.

32. Torgén M, Alfredsson L, Koster M, Wiktorin C, Smith Kf, Kilbom A. Reproducibility of a questionnaire for assessment of a present and past physical activities. Int Arch Occup Environ Health 1997; 70:107-18.

33. Wiktorin C, Wigaeus-Hjelm E, Winkel J, Koster M. Reproducibility of a questionnaire for assessment of physical load during work and leisure time. J Occup Environ Med 1996; 38:190-201.

34. Alipour A, Ghaffari M, Jensen I, Shariati B, Vingard E. Reliability and validity study of Persian modified version of MUSIC (Musculoskeletal Intervention Center) - Norrtalje questionnaire. BMC Musculoskelet Disord 2007; 8:88.

35. Pereira MG. Aferição de eventos. In: Pereira MG, organizador. Epidemiologia: teoria e prática. São Paulo: Editora Guanabara Koogan; 2001. p. 363-76.

36. Sim J, Wright CC. The kappa statistic in reliability studies: use, interpretation, and sample size requirements. Phys Ther 2005; 85:257-68.

37. Speklé E, Hoozemans M, van der Beek A, Blatter B, Bongers P, Can Dieen J. Internal consistency, test-retest reliability and concurrent validity of a questionnaire on work-related exposure related to arm, shoulder and neck symptoms in computer workers. Ergonomics 2009; 52:1087-103. 


\section{Abstract}

This study sought to analyze the dimensionality of a set of items that measure work-related physical demand, their internal consistency and their testretest reliability. We carried out an exploratory factorial analysis of ten physical demand items in two populations, totaling 1,070 workers from the footwear industry and from urban cleaning, using weighted least squares means and adjusted variance with Promax oblique rotation. We measure internal consistency using Cronbach's alpha. The test-retest used a subsample of 121 workers, using quadratic weighted kappa. We identified two dimensions in urban cleaning and three in the footwear industry: (1) dynamic work with anomalous postures; (2) work that requires handling material; and (3) work that demands repetitive motions. Reliability was excellent for upright work posture, good for walking, moderate for lifting loads, squatting, rotated trunk and only regular for repetitive motions and arms above shoulders. The dimensions we identified through factorial analysis are compatible with the physical demands of urban cleaning and the footwear industry and, in general, showed good internal consistency. The 10 items' reliability was compatible with the literature, showing excellent to good reliability for general posture, moderate for specific body segment posture and regular for repetitiveness. Results reveal the specificity of each occupational group, which suggests a dimensionality of physical demand items, supporting their use for measuring physical exposure.

Statistical Factor Analysis; Reproducibility of Results; Workload; Surveys and Questionnaires

\section{Resumen}

El objetivo de este trabajo fue analizar la dimensionalidad de un conjunto de items que mide los requerimientos físicos en el trabajo, su consistencia interna y la confiabilidad test-retest. Se realizó un análisis factorial exploratorio de diez items de requerimientos físicos en dos poblaciones, totalizando 1.070 trabajadores de la industria del calzado y limpieza urbana, utilizando como método de estimación la media de mínimos cuadrados ponderados y variación ajustada, con rotación oblicua Promax. La consistencia interna se midió por el alfa de Cronbach. El test-retest utilizó una submuestra de 121 trabajadores, con la utilización del índice kappa de ponderación cuadrática. Se identificaron dos dimensiones en la limpieza urbana y tres en la fabricación de calzados: (1) trabajo dinámico con posturas anómalas; (2) trabajo que implica manipulación de material; $y$ (3) trabajo con exigencia de movimientos repetitivos. La confiabilidad fue excelente para la postura de trabajo en pie, buena para andando, moderada para levantamiento de carga, trabajo agachado, tronco flexionado y sólo regular para movimientos repetitivos y brazos encima de los hombros. Las dimensiones identificadas en el análisis factorial son compatibles con los requerimientos físicos en limpieza urbana e industria de calzados y, en general, mostraron una buena consistencia interna. La confiabilidad de los diez items fue compatible con la literatura, mostrando como excelente la buena confiabilidad para la postura general, moderada para la postura de un segmento especifico del cuerpo y regular para repetitividad. Los resultados revelan especificidades de cada grupo ocupacional, lo que sugiere la dimensionalidad de los items de requerimientos físicos, apoyando su uso para la medición de la exposición física al trabajo.

Análisis Factorial; Reproducibilidad de los Resultados; Carga de Trabajo; Encuestas y Cuestionários
Recebido em 26/Jun/2018

Versão final reapresentada em 04/Out/2018 Aprovado em 10/Out/2018 